\title{
How the COVID-19 outbreak affected physician-patient relationship
}

\section{Ali Fakhari', Roya Dolatkhah ${ }^{2 *}$ and Keivan Fakhari Dehkharghani ${ }^{3}$}

1Professor of Psychiatry, Research Center of Psychiatry and Behavioral Sciences, Tabriz University of Medical Sciences, Tabriz, Iran

${ }^{2}$ Assistant Professor of Epidemiology, Hematology and Oncology Research Center, Tabriz, Iran ${ }^{3}$ Medical Student, Tabriz, Iran

\section{Abstract}

Physician-patient communication is the most basic element and vital skill of physicians in the diagnosis, treatment, and establishing diagnostic protocols. As the risks of contagion and viral infection are higher in physicians and health workers, front line soldiers inpatient care units, so they should establish strict protective measures. However, patients value greatly face to face and close relationships with their doctors, including facial interviews and physical examinations. So social and physical distancing between physician and their patients can be remarked as a bigger toll than the risk of COVID-19 contagious.

\section{Introduction}

The pandemic outbreak has caused many difficulties and significant damage to health, economy, and human relations. It has transformed our world and brought about fundamental changes in human relations and modern civilization.

In addition to these effects, physicians and health care providers have been the most exposed and have experienced more mental harm than others [1,2]. Undoubtedly the most basic and important results of these effects are the potential and actual risk of exposure to infected patients and the risks of transmission of this disease. The contagion rate and mortality of physicians and healthcare providers engaged in patient care, especially in COVID-19 intensive care units, is clear [3].

Physician-patient communication is the most basic element and vital skill of physicians in the diagnosis, treatment, and establishing diagnostic protocols. With good communication, physicians can build a safe, scientific, practical, and suitable environment for patient care [4]. To establish this environment, the main principles include:

1 Building a proper area for patient care

2 Proficiency in setting, organizing, and practicing good physician-patient communication

3 Providing useful feedback to the patient

However, many things have changed for about $77 \%$ of

\section{In-patient care units}

More Information

*Address for Correspondence: Roya Dolatkhah, Hematology and Oncology Research Center, Tabriz University of Medical Sciences, Tabriz, Iran, Tel/Fax: +98 4133361358;

Email: royadolatkhah@yahoo.com

Submitted: 30 October 2020

Approved: 16 November 2020

Published: 17 November 2020

How to cite this article: Fakhari $A$,

Dolatkhah R, Dehkharghani KF. How the COVID-19 outbreak affected physician-patient relationship. J Community Med Health Solut. 2020; 1: 023-025.

DOI: 10.29328/journal.jcmhs. 1001003

ORCiD: orcid.org/0000-0002-6897-7120

Copyright: (c) 2020 Fakhari A, et al. This is an open access article distributed under the Creative Commons Attribution License, which permits unrestricted use, distribution, and reproduction in any medium, provided the original work is properly cited.

Keywords: COVID-19; Physician; Patient; Relationship; Outbreak

Check for updates

OPEN ACCESS

physicians and nearly $3 / 4$ of nurses and healthcare workers. Health care workers establish these conditions differently for inpatient and outpatient care units.

In COVID-19 care units, as the risks of contagion and viral infection are higher, physicians and health workers should establish strict protective measures. These include face masks, face shields, and special protective coats; these are known as PPE (Personal Protective Equipment). This enables physicians and patients to be completely physically isolated from each other. This is not a problem in an intensive care unit (ICU) where most of the patients are unconscious, but in other patient care units, physicians and health care workers earning protective measures can be traumatic for the patient and may lead to emotional relationship disruption [5].

Interruption in conversation, face-to-face emotional disconnection, and incomprehensible bilateral reaction increase this dysfunction in communication. Additional communication difficulties include speech and listening impairment, and impersonal connection due to the face mask. This further disrupts the ideal emotional relationship between physicians and patients. The fear and anxiety in the care units, worsening of patients' conditions, and deaths lead to further deterioration of the hospital and/or care unit 
environment. This deterioration is evident and different on both patient and physician sides. The patient has the fear and stress of being marked with a dangerous and untreatable disease as well as their physical condition. Being in physical quarantine from family and friends, having a serious respiratory disease, and fear of mortality ultimately lead to traumatic and painful psychosocial status.

Physicians and health care workers have different emotional reactions. Undoubtedly one of the most important aims of physicians is patients' comprehensive medical support and in this way, they use all of the available treatments, diagnostic tools, and interventions even if they suffer serious complications; but there were different circumstances during this pandemic. This new disease is almost unknown, and there is a lot of ambiguity about its pathophysiology, contagion methods and risks, symptoms, and treatment efforts, so these lead to severe ambivalence and confusion in physicians and healthcare workers [6-8]. Although they use all their skills and abilities for patient care, the fear of contagion, and being quarantined from family leads to their additional physiological distress. They have fear and anxiety about exposure to COVID-19 at work and bringing the infection home to family $[6,9]$. However the self-sacrifice and confidence of healthcare workers around the world are unforgettable; certainly, the value of this professional courage and sacrifice will never diminish.

\section{Out-patient care units}

Outpatient care units include an entirely different environment that offers different problems and events. The most important recent events and issues are:

- Impairment of the activities of several unnecessary outpatient physicians

- Prohibition of physicians' high-risk activities by health systems and/or governments

- Refraining from outpatient activities by some physicians

- Continuation to normal work procedures by some physicians

Regardless of the above issues, there were other serious challenges for outpatient care physicians:

- How to manage and make appointments

- How to manage waiting lists and office attendance

- How to work with patients in the examination room

- Whether or not to use online tools for patient visits

Online visits, contact using social media, and telemedicine services are embraced in some patient care units during the current pandemic. However, most patients are not willing to continue this practice. Moreover, the physician/patient relationship and their communication are somehow different from other diseases, and patients who are referred to psychologists and/or psychiatrists prefer to have unrestricted in-person visits without facial masks and social distancing. All previous surveys emphasize that face-to-face and personal interplay is most valuable for patients, rather than social and physical distancing in the current circumstances [10].

Physicians feared that a tsunami of patients would overwhelm their healthcare systems, preventing them from adequately caring for other patients and/or managing their own contagion risk [1]. Meanwhile, an accumulation of patients in the waiting room creates a dangerous environment for the possibility of spreading the disease. Following social distancing protocols, managing patients' appointments, and avoiding crowded offices and waiting rooms are observed in most outpatient clinics. However, because of severe fear and anxiety of contagion requiring physical barriers and plastic shields, the relationships between office receptionists and patients become problematic. The most important disruption occurred in the waiting room. The positive attitude of patients and physicians is observed in "Field Observations". Lack of face-to-face interactions lead to a less human practice of medicine, while patient-physician relationships in most outpatient clinics are based on communication, collaboration, trust, and empathy between physicians and patients [11].

Patients value greatly the face to face and close relationships with their doctors, including facial interviews and physical examination. So social and physical distancing between physician and their patients can be remarked as a bigger toll than the risk of COVID-19 contagious [11].

Doctors, on the other hand, have serious psychological conflicts between commitment to care and the risk of contagiousness of the disease. Numerous field observations and reports indicate a very diverse behavior of physicians in this regard. Some physicians (even internal medicine and cardiologists) visit their patients with face mask and shield and with physical distancing. Some of them even refused to clinical and physical examination this was accurate even for the control of vital signs such as measuring the patient's blood pressure. Some doctors had a major problem with the face mask during the interview with the patient. This was more problematic in clinics where verbal history is very important. Having a mask had a profound effect on how the doctor and the patient spoke and listened to each other, this was most problematic when the patients have masks too. This problem is most obvious when using the N95 mask, because of confusing echo sounds during the speaking. Some patients have to remove their face mask during speaking because they think that maybe physicians can't understand them so many doctors complain, and this leads to disruption in their communications. Awareness of reciprocal facial changes, 
which is one of the basic principles of physician-patient communication, has been strongly influenced, and psychiatric interviews have been used to remove the emotion or affect the index from the psychiatric description. The patient's approach to presenting medical records to the physician has sometimes been accompanied by a serious reaction from the physician, followed by a disturbance in the patient's physician relationship.

\section{Conclusion}

In conclusion, the recent outbreak is harmful sophisticated conditions, while physicians and health care workers are "The soldiers on the front lines". Even sometimes these front line soldiers didn't access appropriate PPE. Meanwhile, infected doctors and nurses have been recognized as "vector for the spread of the virus", and for those infected health care workers, the feeling of powerlessness is so painful.

However, the most important positive side of this circumstance is reminding and awareness of the most general population about professional physicians> roles and their commitment to providing care even by accepting the risks of contagious. It was obvious from the reaction of ordinary people, officials, and journalists on social media and TV channels. Occasionally, different ceremonies and reactions taking care of health care workers were particularly emotional.

\section{Acknowledgement}

\section{Authorship}

(1) AF: performed the conception and design of the study, and acquisition of data.

(2) $\mathrm{AF}, \mathrm{RD}, \mathrm{KFD}$ : performed drafting the article and revising it critically for important intellectual content.

(3) $\mathrm{AF}, \mathrm{RD}$, and KFD: approved the version to be submitted.

\section{References}

1. COVID-19 Is Making Moral Injury to Physicians Much Worse. Medscape Business of Medicine. 2020.

PubMed: https://www.medscape.com/viewarticle/927859.

2. Sun $N$, Wei $L$, Shi $S$, Jiao $D$, Song $R$, et al. A qualitative study on the psychological experience of caregivers of COVID-19 patients. Am J Infect Control. 2020; 48: 592-598.

PubMed: https://pubmed.ncbi.nIm.nih.gov/32334904/

3. Alikhani R, Salimi A, Hormati A, Aminnejad R. Mental health advice for frontline healthcare providers caring for patients with COVID-19. Can J Anaesth. 2020; 67: 1068-1069.

PubMed: https://pubmed.ncbi.nlm.nih.gov/32291624/

4. Luykx JJ, Vinkers CH, Tijdink JK. Psychiatry in Times of the Coronavirus Disease 2019 (COVID-19) Pandemic: An Imperative for Psychiatrists to Act Now. JAMA Psychiatry. 2020.

PubMed: https://pubmed.ncbi.nlm.nih.gov/32459359/

5. Gaeta C, Brennessel R. COVID-19: Emergency Medicine Physician Empowered to Shape Perspectives on This Public Health Crisis. Cureus. 2020; 12: e7504.

PubMed: https://pubmed.ncbi.nlm.nih.gov/32257730/

6. El-Hage $W$, Hingray $C$, Lemogne $C$, Yrondi A, Brunault $P$, et al Health professionals facing the coronavirus disease 2019 (COVID-19) pandemic: What are the mental health risks?. Encephale. 2020; 46: S73-S80.

PubMed: https://pubmed.ncbi.nlm.nih.gov/32370984/

7. Iserson KV. Healthcare Ethics During a Pandemic. West J Emerg Med. 2020; 21: 477-483.

PubMed: https://www.ncbi.nlm.nih.gov/pmc/articles/PMC7234717/

8. Hayry M. The COVID-19 Pandemic: Healthcare Crisis Leadership as Ethics Communication. Camb Q Healthc Ethics. 2020: 1-9.

PubMed: https://pubmed.ncbi.nlm.nih.gov/32438949/

9. Restauri N, Sheridan AD. Burnout and Posttraumatic Stress Disorder in the Coronavirus Disease 2019 (COVID-19) Pandemic: Intersection, Impact, and Interventions. J Am Coll Radiol. 2020; 17: 921-926. PubMed: https://pubmed.ncbi.nIm.nih.gov/32479798/

10. Serper M, Cubell AW, Deleener ME, Casher TK, Rosenberg DJ, et al. Telemedicine in Liver Disease and Beyond: Can the COVID-19 Crisis Lead to Action? Hepatology. 2020; 72: 723-728.

PubMed: https://pubmed.ncbi.nlm.nih.gov/32275784/

11. Rodler S, Apfelbeck M, Schulz GB, Ivanova T, Buchner A, et al. Telehealth in Uro-oncology Beyond the Pandemic: Toll or Lifesaver? Eur Urol Focus. 2020; 6: 1097-1103.

PubMed: https://pubmed.ncbi.nlm.nih.gov/32534969/ 